\title{
Microscopic versus Endoscopic Inlay Butterfly Cartilage Tympanoplasty
}

\author{
Se A Lee, Hyun Tag Kang, Yun Ji Lee, Bo Gyung Kim, and Jong Dae Lee \\ Department of Otorhinolaryngology-Head and Neck Surgery, Soonchunhyang University Bucheon Hospital, Bucheon, Korea
}

Received December 31, 2018

Revised February 8, 2019

Accepted February 27, 2019

\author{
Address for correspondence \\ Jong Dae Lee, MD, PhD \\ Department of Otorhinolaryngology- \\ Head and Neck Surgery, \\ Soonchunhyang University \\ Bucheon Hospital, 170 Jomaru-ro, \\ Bucheon 14584, Korea \\ Tel $+82-32-621-5015$ \\ Fax +82-32-621-5016 \\ E-mail1jdent@schmc.ac.kr
}

Background and Objectives: Inlay butterfly cartilage tympanoplasty makes the graft easy, and reduces operating time. The present study aimed to investigate the outcomes of microscopic versus endoscopic inlay butterfly cartilage tympanoplasty. Subjects and Methods: In this retrospective study, the outcomes of 63 patients who underwent inlay butterfly cartilage tympanoplasty with small to medium chronic tympanic membrane perforation were evaluated. Twenty-four patients underwent conventional microscopic tympanoplasty and 39 underwent endoscopic tympanoplasty. The outcomes were analyzed in terms of the hearing gain and graft success rate. Results: The surgical success rate was $95.8 \%$ in the patients who underwent conventional microscopic tympanoplasty and $92.3 \%$ in those who underwent endoscopic tympanoplasty. In both groups of patients, the postoperative air-bone gap (ABG) was significantly lower than the preoperative ABG. There were no significant differences between the preoperative and postoperative ABG values in either group. Conclusions: Endoscopic inlay tympanoplasty using the butterfly cartilage technique appears to be an effective alternative to microscopic tympanoplasty and results in excellent hearing.

J Audiol Otol 2019;23(3):140-144

KEY WORDS: Endoscopy · Microscopy · Tympanoplasty · Tympanic membrance perforation.

\section{Introduction}

Since Zollner [1] and Wullstein [2] introduced tympanoplasty in the 1950s, many techniques and graft materials have been developed to close perforations of the tympanic membrane. Inlay butterfly cartilage tympanoplasty was first described by Eavey in 1998 [3]. Tragal cartilage is used as the graft and the surgeon inserts the cartilage graft within the perforated edges via the external ear canal. The butterfly technique does not require elevation of a tympanomeatal flap, so no canal incision or postoperative packing is necessary.

Middle ear surgery was traditionally performed under a microscope. However, the endoscope was started to be used in ear surgery in the 1990s [4], and since then has become popular with surgeons. The surgical field is limited using the transcanal approach with a microscope, whereas the endoscopic

This is an Open Access article distributed under the terms of the Creative Commons Attribution Non-Commercial License (https://creativecommons.org/licenses/by-nc/4.0/) which permits unrestricted non-commercial use, distribution, and reproduction in any medium, provided the original work is properly cited. approach allows good visualization of the middle ear cavity $[5,6]$.

Several studies have compared microscopic tympanoplasty and endoscopic tympanoplasty [7-9], but few have compared microscopic and endoscopic inlay tympanoplasty using the butterfly cartilage technique. The aim of this study was to investigate the outcomes of endoscopic versus microscopic inlay butterfly cartilage tympanoplasty.

\section{Subjects and Methods}

\section{Subjects}

We reviewed the medical records of patients who underwent inlay butterfly cartilage tympanoplasty to treat small or medium chronic tympanic membrane perforation in the Department of Otorhinolaryngology-Head and Neck Surgery at Soonchunhyang University Bucheon Hospital. Patients who underwent conventional microscopic tympanoplasty from March 2011 to August 2014 were enrolled in a microscopic tympanoplasty group and those who underwent endoscopic 
tympanoplasty from September 2015 to February 2018 were enrolled in an endoscopic tympanoplasty group. Computed tomographic scans of the temporal bone were obtained in all cases to evaluate the status of the middle ear and mastoid cavity. The outcomes were analyzed in terms of graft success rate and gain in hearing. The study was approved by the Institutional Review Board of Soonchunhyang University (IRB No.: 2018-07-002).

\section{Surgical technique}

The same surgeon (JDL) performed all the inlay butterfly cartilage tympanoplasty procedures under local anesthesia using a transcanal approach. The margin of the tympanic membrane was freshened circumferentially by trimming with a sharp pick.

Tragal cartilage was used as the graft material in all cases. A skin incision was made on the medial side of the tragal cartilage. The cartilage graft was shaped to be $2 \mathrm{~mm}$ wider in diameter than the perforation. The graft was incised all along its edge, forming a "butterfly" wing shape, and the perichondrium of the outer surface of the cartilage was removed. The cartilage graft was then inserted into the perforation using an inlay technique so that its perichondrium-free surface was facing towards the middle ear (Fig. 1).

A microscope (Carl Zeiss, Oberkochen, Germany) was used in the microscopic tympanoplasty group and a 0-degree rigid endoscope (3 mm diameter, $14 \mathrm{~cm}$ long; Karl Storz, Tuttlingen, Germany) and high definition monitor and camera (Karl Storz, Tuttlingen, Germany) were used in the endoscopic tympanoplasty group.

\section{Postoperative outcome}

The tympanic membrane and mastoid cavity were initially examined one week after the surgery using a surgical microscope. The patients were then followed up at 1 month postoperatively and at 3-month intervals for 6 months. Hearing outcomes were evaluated by comparing the air-bone gap $(\mathrm{ABG})$ values and the average hearing threshold at four frequencies $(500,1,000,2,000$, and 4,000 Hz) before and 6 months after surgery. The success of the surgery was defined as the complete closure of the tympanic membrane perforation without any signs of infection at 6 months after the operation.

\section{Statistical analysis}

Differences in the hearing thresholds between the conventional microscopic tympanoplasty group and the endoscopic tympanoplasty group were evaluated using the Mann-Whitney U test and Fisher's exact test. A $p$-value $<0.05$ was considered statistically significant. The statistical analysis was performed using SPSS version 20.0 software (IBM Corp.,

Fig. 1. The tragal cartilage myringoplasty technique. (A) A cartilage graft is obtained from the tragal cartilage. (B) Make a groove circumferentially with a thin edge of the cartilage graft to make the shape of a butterfly. The perichondrium is elevated and removed on the external auditory canal side of the cartilage; (C) the cartilage graft is pushed through the perforation. (D) The cartilage graft is placed in an inlay fashion with its perichondrium-free surface facing towards the middle ear.
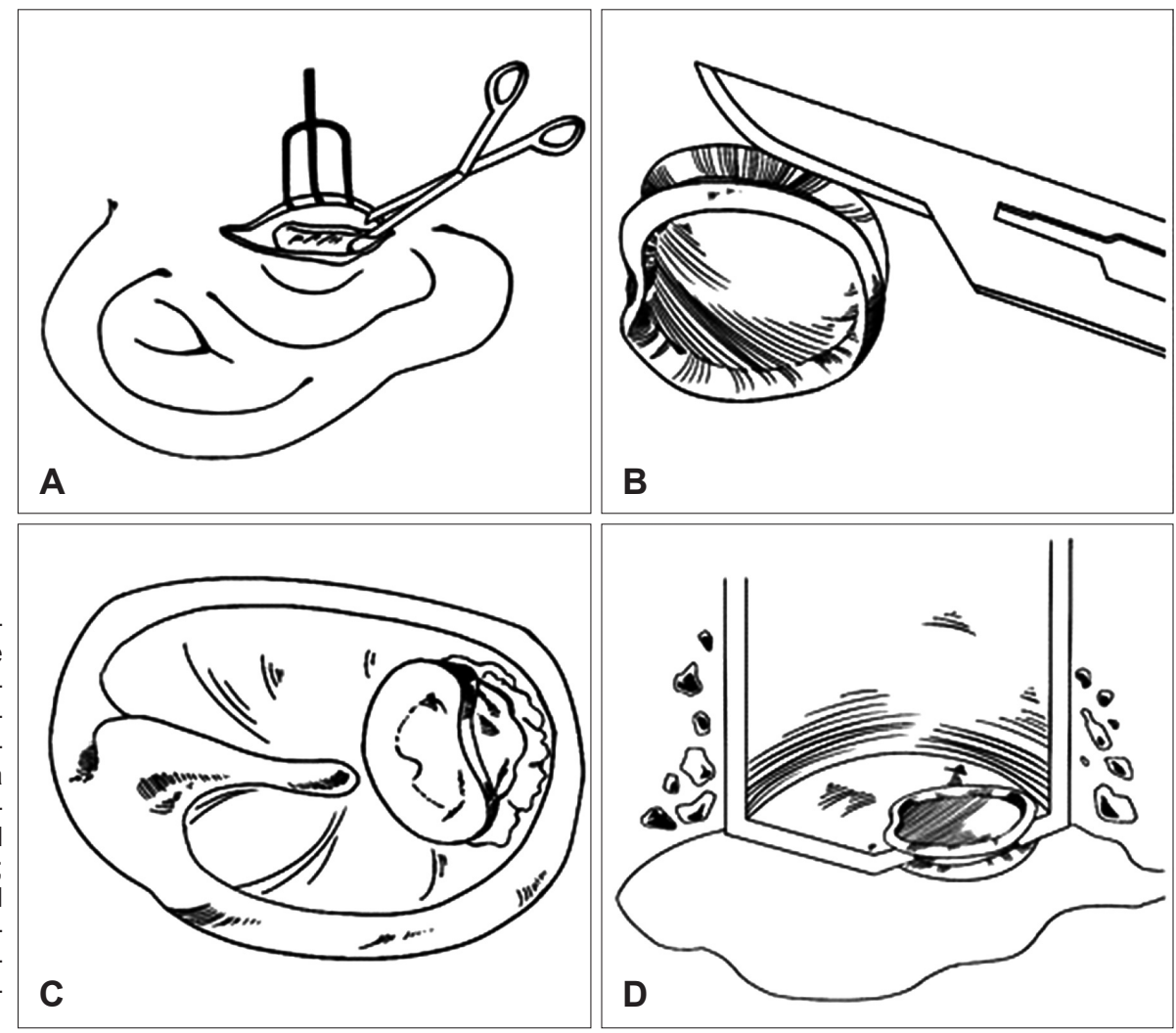
Armonk, NY, USA).

\section{Results}

Demographic information for all 63 patients in the study is summarized in Table 1. Twenty-four patients (12 male, 12 female) underwent conventional microscopic tympanoplasty and 39 patients (13 male, 26 female) underwent endoscopic tympanoplasty. There was no significant difference in age, size, or anatomic location of the perforation between the two groups.

Fig. 2 shows the views under microscopy and endoscopy. The anterior edge of the perforation was not visible under a microscope. However, the endoscopic view revealed an image of the entire tympanic membrane in one field and the perforation edge could be clearly visualized even when the ear canal was narrow and/or protruded (Supplementary Video 1 in the online-only Data Supplement, which compares the sur-

Table 1. Demographic and clinical characteristics of the patients

\begin{tabular}{|c|c|c|c|}
\hline & $\begin{array}{l}\text { Microscopic } \\
\text { tympanoplasty }\end{array}$ & $\begin{array}{l}\text { Endoscopic } \\
\text { tympanoplasty }\end{array}$ & $\begin{array}{c}\mathrm{p}- \\
\text { value }\end{array}$ \\
\hline Number of patients & 24 & 39 & \\
\hline Male:Female & $12: 12$ & $13: 26$ & 0.189 \\
\hline Mean age (years) & $53.04 \pm 16.31$ & $55.10 \pm 16.20$ & 0.627 \\
\hline Size of perforation (n) & & & 0.881 \\
\hline $2 \mathrm{~mm}$ & 11 & 20 & \\
\hline $3 \mathrm{~mm}$ & 9 & 14 & \\
\hline $4 \mathrm{~mm}$ & 4 & 5 & \\
\hline $\begin{array}{l}\text { Location of } \\
\text { perforation (n) }\end{array}$ & & & 0.164 \\
\hline Central & 16 & 19 & \\
\hline Anterior & 8 & 20 & \\
\hline Operating time (min) & $35.20 \pm 12.20$ & $31.87 \pm 12.59$ & 0.305 \\
\hline
\end{tabular}

Values are presented as mean \pm standard deviation unless otherwise indicated. gical view under the microscope with that via an endoscope).

There was no statistically significant difference in the actual operative time between microscopic tympanoplasty group $(35.20 \pm 12.20 \mathrm{~min})$ and endoscopic tympanoplasty group $(31.87 \pm 12.59 \mathrm{~min})(p=0.305)$.

The success rate was $95.8 \%(23 / 24)$ in the microscopic tympanoplasty group and $92.3 \%(36 / 39)$ in the endoscopic tympanoplasty group; the success rate was not significantly different between the two groups. The tympanoplasty procedure failed in four patients (one in microscopic tympanoplasty group and three in endoscopic tympanoplasty group) and the perforation recurred.

There was no statistically significant difference in the mean preoperative $\mathrm{ABG}$ between the microscopic tympanoplasty group and the endoscopic tympanoplasty group $(8.59 \pm 8.01$ $\mathrm{dB}$ vs. $8.82 \pm 4.63 \mathrm{~dB})$ or in the postoperative ABG (3.32 \pm $4.67 \mathrm{~dB}$ vs. $3.09 \pm 3.82 \mathrm{~dB}$ ). The difference between the preoperative and postoperative ABG was analyzed in each study group using the paired t-test; the postoperative ABG was significantly lower than the preoperative $\mathrm{ABG}$ in both groups $(p<0.05)$. There was no statistically significant difference in the mean improvement in ABG between the microscopic tympanoplasty group and the endoscopic tympanoplasty group (5.27 $\pm 6.90 \mathrm{~dB}$ vs. $5.73 \pm 5.37 \mathrm{~dB}, p=0.776$; Fig. 3).

\section{Discussion}

Since Eavey decribed the inlay butterfly cartilage tympanoplasty in 1998, many studies about inlay butterfly cartilage technique have been introduced and they have used "myringoplasty" and "tympanoplasty" without distinction [3,10-12]. So we used the term inlay butterfly cartilage tympanoplasty as a proper noun.

Surgeons have been reporting good results from inlay butterfly cartilage tympanoplasty since its introduction by Eavey
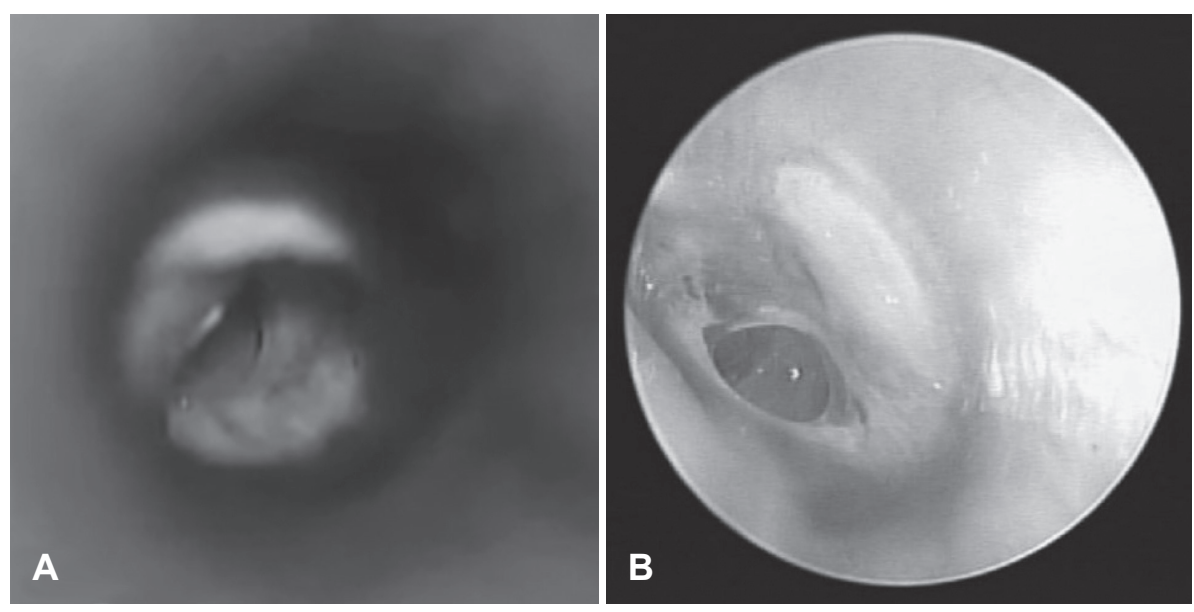

Fig. 2. Surgical view of anterior tympanic membrane perforation. (A) Microscopic view. (B) Endoscopic view. 


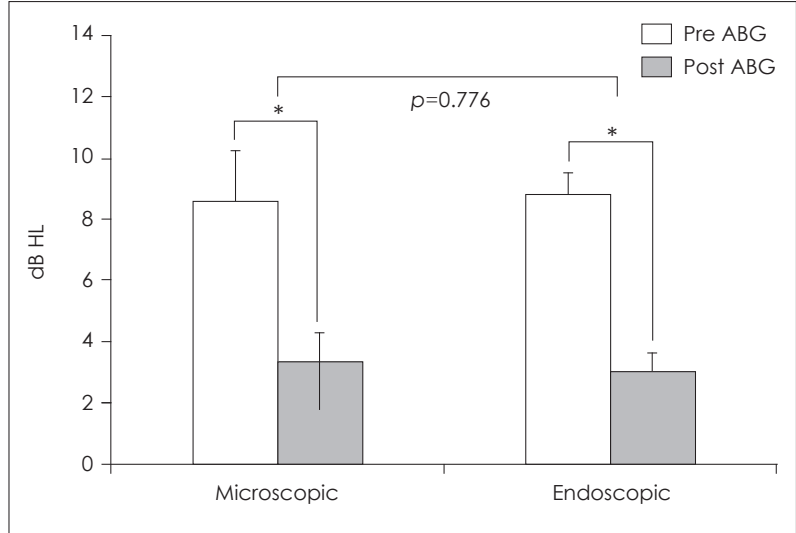

Fig. 3. Comparision between preoperative and postoperative airbone gap (ABG). There was no statistically significant difference in the mean improvement in ABG between the microscopic tympanoplasty group and the endoscopic tympanoplasty group. ${ }^{*} p<$ 0.05. dB HL: decibel hearing level (bars represent standard error of the mean).

[3] 20 years ago. Ghanem, et al. [10] reported a $92 \%$ success rate for perforations of medium to large size and Kim, et al. [11] reported a $96.4 \%$ success rate for perforations of any size. Moreover, good results have been reported using endoscopic inlay butterfly cartilage tympanoplasty, with Özgür, et al. [12] reporting a graft success rate of $95.6 \%$ and Akyigit, et al. [13] reporting intact grafts in $93.7 \%$ of children who underwent an endoscopic procedure. Inlay butterfly cartilage tympanoplasty is feasible, safe, and efficient, and does not require a tympanomeatal flap or ear packing. These advantages make inlay butterfly cartilage tympanoplasty an attractive surgical option for surgeons. In the present study, we compared the results of microscopic and endoscopic inlay butterfly cartilage tympanoplasty. There was no significant difference in the success rate between microscopic tympanoplasty and endoscopic tympanoplasty (95.8\% vs. $92.3 \%)$. There were four failed cases in which perforations recurred in the same position; there was no otorrhea at the time of surgery in these patients but all had a history of otorrhea. Therefore, it is worth discussing the possibility of failure with patients who have a history of otorrhea.

Kuo and $\mathrm{Wu}[8]$ reported that the endoscopic operating time was shorter than the microscopic operating time (74.4 vs. $101.9 \mathrm{~min}, p$-value $<0.001$ ). In our study, there was no significant difference in operating time between microscopic tympanoplasty and endoscopic tympanoplasty. We performed the inlay butterfly cartilage tympanoplasty procedures and only incision was made on tragus without tympanomeatal flap elevation. Because the own operating time of the inlay butterfly cartilage tympanoplasty is shorter than underlay tympanoplasty, there was no difference between the microscopic and endoscopic tympanoplasty group.
There has been concern regarding using cartilage as a graft material because of the rigidity of the cartilage [14]. However, some investigators have reported finding no difference in postoperative hearing results between cartilage tympanoplasty and perichondrium or temporalis fascia tympanoplasty $[11,15,16]$. Furthermore, there is no difference in hearing results between microscopic and endoscopic tympanoplasty. Lade, et al. [9] and Kuo and $\mathrm{Wu}$ [8] compared the improvement in hearing between a microscopic group and an endoscopic group and found no significant difference between them. In the present study, the difference between the preoperative $\mathrm{ABG}$ and postoperative $\mathrm{ABG}$ was analyzed and the postoperative $\mathrm{ABG}$ was significantly lower than the preoperative $\mathrm{ABG}$ in both groups. However, the improvement in $\mathrm{ABG}$ was not significantly different between the two groups. It is probable that the amount of hearing improvement after tympanoplasty depends on the success of the graft, any complications that occur, and recurrence of perforation rather than the choice of approach or use of a microscope or endoscope.

Anterior perforations of the tympanic membrane are the most challenging to repair. We need to adjust the microscope further or turn the patient's head more toward the opposite side. When there is anterior bony overhang or a narrow anterior tympanomeatal angle, it is almost impossible to observe perforation through an ear speculum. We find it difficult to perform inlay butterfly cartilage tympanoplasty for an anterior perforation under a microscope; however, an endoscopic approach solves this problem.

In summary, endoscopic inlay butterfly cartilage tympanoplasty appears to be an effective alternative to microscopic tympanoplasty and results in excellent hearing. An endoscopic procedure may be a reasonable option for patients with a narrow external ear canal, anterior perforation, or bony overhang. Further studies in large patient population are needed to investigate.

\section{Supplementary Video}

Video 1. Comparison between the surgical view under the microscope and that via an endoscope.

\section{Supplementary Materials}

The online-only Data Supplement is available with this article at https://doi.org/10.7874/jao.2018.00549.

\section{Acknowledgments}

This study was supported by the Soonchunhyang University Research Fund.

\section{Conflicts of interest}

The authors have no financial conflicts of interest. 


\section{Author Contributions}

Conceptualization: Jong Dae Lee. Data curation: Se A Lee, Hyun Tag Kang, Yun Ji Lee, and Bo Gyung Kim. Formal analysis: Se A Lee. Methodology: Jong Dae Lee. Project administration: Se A Lee. Resources: Jong Dae Lee. Software: Se A Lee. Supervision: Jong Dae Lee. Validation: Se A Lee and Jong Dae Lee. Visualization: Se A Lee. Writing — original drift: Se A Lee. Writing — review \& editing: Se A Lee and Jong Dae Lee.

\section{ORCID iDs}

\section{Se A Lee}

Hyun Tag Kang

Yun Ji Lee

Bo Gyung Kim

Jong Dae Lee

\section{REFERENCES}

1) Zollner F. The principles of plastic surgery of the sound-conducting apparatus. J Laryngol Otol 1955;69:637-52.

2) Wullstein $H$. Theory and practice of tympanoplasty. Laryngoscope 1956;66:1076-93.

3) Eavey RD. Inlay tympanoplasty: cartilage butterfly technique. Laryngoscope 1998;108:657-61.

4) Thomassin JM, Duchon-Doris JM, Emram B, Rud C, Conciatori J, Vilcoq P. Endoscopic ear surgery. Initial evaluation. Ann Otolaryngol Chir Cervicofac 1990;107:564-70.

5) Karhuketo TS, Laippala PJ, Puhakka HJ, Sipilä MM. Endoscopy and otomicroscopy in the estimation of middle ear structures. Acta

Otolaryngol 1997;117:585-9.

6) Karhuketo TS, Puhakka HJ, Laippala PJ. Endoscopy of the middle ear structures. Acta Otolaryngol Suppl 1997;529:34-9.

7) Choi N, Noh Y, Park W, Lee JJ, Yook S, Choi JE, et al. Comparison of endoscopic tympanoplasty to microscopic tympanoplasty. Clin Exp Otorhinolaryngol 2017;10:44-9.

8) Kuo CH, Wu HM. Comparison of endoscopic and microscopic tympanoplasty. Eur Arch Otorhinolaryngol 2017;274:2727-32.

9) Lade H, Choudhary SR, Vashishth A. Endoscopic vs microscopic myringoplasty: a different perspective. Eur Arch Otorhinolaryngol 2014;271:1897-902.

10) Ghanem MA, Monroy A, Alizade FS, Nicolau Y, Eavey RD. Butterfly cartilage graft inlay tympanoplasty for large perforations. Laryngoscope 2006;116:1813-6.

11) Kim HJ, Kim MJ, Jeon JH, Kim JM, Moon IS, Lee WS. Functional and practical outcomes of inlay butterfly cartilage tympanoplasty. Otol Neurotol 2014;35:1458-62.

12) Özgür A, Dursun E, Terzi S, Erdivanlı ÖÇ, Coşkun ZÖ, Oğurlu M, et al. Endoscopic butterfly cartilage myringoplasty. Acta Otolaryngol 2016;136:144-8.

13) Akyigit A, Karlidag T, Keles E, Kaygusuz I, Yalcin S, Polat C, et al. Endoscopic cartilage butterfly myringoplasty in children. Auris Nasus Larynx 2017;44:152-5.

14) Lubianca-Neto JF. Inlay butterfly cartilage tympanoplasty (Eavey technique) modified for adults. Otolaryngol Head Neck Surg 2000; 123:492-4.

15) Dornhoffer JL. Hearing results with cartilage tympanoplasty. Laryngoscope 1997;107:1094-9.

16) Gerber MJ, Mason JC, Lambert PR. Hearing results after primary cartilage tympanoplasty. Laryngoscope 2000;110:1994-9. 